\title{
Mediastinal Choriocarcinoma Masquerading as Relapsed Hodgkin Lymphoma
}

\author{
Selay Lam ${ }^{a}$ Kamilia Rizkalla ${ }^{b}$ Cyrus C. Hsia \\ ${ }^{a}$ Division of Haematology, Department of Medicine and ${ }^{b}$ Department of \\ Pathology, London Health Sciences Centre, London, Ont., Canada
}

\section{Key Words}

Germ cell tumour $\cdot$ Choriocarcinoma $\cdot$ Hodgkin lymphoma $\cdot$ Mediastinal tumour

\begin{abstract}
Primary mediastinal choriocarcinoma is a rare extragonadal germ cell malignancy. We describe the first case of a patient who developed mediastinal choriocarcinoma after treatment for Hodgkin lymphoma (HL). A 25-year-old man with classic HL, nodular sclerosis subtype, underwent treatment with splenectomy followed by radiation therapy. Unfortunately, his disease relapsed with a paraspinal mass, and he was subsequently treated with MOPP (mechlorethamine, Oncovin, procarbazine, and prednisone) alternating with ABVD (Adriamycin, bleomycin, vinblastine, and dacarbazine). He achieved a complete remission after 6 cycles. Ten years after treatment, the patient presented with a persistent cough, haemoptysis, right supraclavicular lymphadenopathy, and weight loss. His chest X-ray showed opacification of the lower right hemithorax with a widened mediastinum. Given unresponsiveness to several antibiotics and lack of evidence for lung volume loss, there were concerns over lung infiltration with relapsed lymphoma. Transbronchial fine needle aspiration biopsy suggested recurrence of his HL. MOPP alternating with ABVD was again given. Due to disease progression, brachytherapy as well as a cocktail of dexamethasone, cytarabine, and cisplatin were also tried. However, on a subsequent excisional lymph node biopsy, it turned out that the tumour was in fact choriocarcinoma and not relapsed HL. Unfortunately, despite aggressive therapy, the patient's disease rapidly progressed, and he died within 2 weeks.
\end{abstract}

\section{Introduction}

Germ cell cancer occurs relatively infrequently, but it is one of the most common malignancies in men aged 15-35 years. It typically originates in the gonads. Extragonadal germ cell tumours, which account for only 2-6\% of all germ cell cancer [1], tend to arise from the midline of the body, namely the pineal gland [2], 
mediastinum [3], retroperitoneum [4], lung parenchyma [5], and sacrococcygeal region. The mediastinum is the most common of the extragonadal sites [6]. In a previous analysis [7] of 322 cases, it was found that nonteratomatous germ cell tumours accounted for $65 \%$ of primary germ cell tumours of the mediastinum, with only $4.3 \%$ of them being choriocarcinoma. The vast majority of them were found to have advanced systemic involvement.

It is unknown what the underlying cause or specific risk factors are for the development of mediastinal choriocarcinoma. We describe, to our knowledge, the first case of mediastinal choriocarcinoma masquerading as relapsed Hodgkin lymphoma (HL).

\section{Case Description}

A 25-year-old man presenting with left supraclavicular lymphadenopathy and suspicious paraaortic nodes underwent a diagnostic staging laparotomy. He was found to have only focal areas of tumour involvement in the spleen, and on biopsy of the involved tissue a diagnosis of nodular sclerosing HL was made. He had stage IIIA disease and was successfully treated with splenectomy along with mantle, para-aortic, and splenic bed irradiation.

Approximately 6 years later, he developed a prolonged history of intermittent fevers, headache, flushing, palpitation, and flank and back pain with no neurological symptoms or signs. He was found to have a large extradural and paraspinal mass at the level of T11-T12 without leptomeningeal involvement. This extradural tumour was resected, and pathology was consistent with relapsed nodular sclerosing HL. He was subsequently treated with chemotherapy consisting of 6 cycles of MOPP (mechlorethamine, Oncovin, procarbazine, and prednisone) alternating with ABVD (Adriamycin, bleomycin, vinblastine, and dacarbazine). A complete remission was achieved.

Ten years after treatment, he presented with several weeks of fatigue, persistent cough, haemoptysis, pleurisy, and weight loss. A right supraclavicular lymph node persisted despite multiple courses of antibiotics for treatment of suspected pneumonia. Serial chest X-rays revealed an enlarging right lower lobe opacity, right paratracheal adenopathy, and widening of his right anterior mediastinum. Transbronchial aspiration initially suggested recurrence of his HL, and he was subsequently started on MOPP alternating with ABVD again.

Unfortunately, a month after he received 1 cycle of chemotherapy, our patient was hospitalised for progressive dyspnea and found to have an enlarging large right-sided pleural effusion. While he was hospitalised, a chest tube was inserted, and an excisional biopsy of a right supraclavicular node was obtained. The patient was treated with multiple courses of brachytherapy via bronchoscopy. However, his condition rapidly deteriorated with clinical evidence of left upper lobe opacity and pericardial effusion without cardiac tamponade. He was transferred to the intensive care unit for mixed hypoxic/hypercapnic respiratory failure requiring mechanical ventilation. Due to extensive tumour invasion visualised by bronchoscopy, he was urgently treated with a cocktail of dexamethasone, cytarabine, and cisplatin, also known as DHAP, as well as concurrent therapy for a potential pulmonary embolus and possible superimposed pneumonia.

After completion of immunohistochemical studies, the diagnosis of choriocarcinoma was confirmed. Unfortunately, the patient's disease further progressed, and he ultimately died of respiratory failure despite mechanical ventilation. The autopsy confirmed the diagnosis of choriocarcinoma with multiple masses involving the mediastinum (measuring $10 \times 5 \times 5 \mathrm{~cm}$ ), all lobes of the right lung $(15 \times 10 \times 8 \mathrm{~cm})$, the right diaphragm, parietal pleura, pericardium, and the upper lobe of the left lung. No testicular involvement was identified. 


\section{Discussion}

Histologically, choriocarcinoma typically consists of a mixed proliferation of syncytiotrophoblasts (large, atypical multinucleated cells with bizarre nuclei, prominent nucleoli, and abundant eosinophilic cytoplasm) and cytotrophoblasts (characterised by their uniform, round nuclei with clear cytoplasm, prominent nucleoli, and numerous atypical mitoses), as previously described [8]. The tumour tends to include haemorrhagic and necrotic components due to its rapid growth. Immunohistochemically, the cells stain strongly positive for keratin and beta-human chorionic gonadotropin.

In contrast, nodular sclerosing HL encompasses the presence of classic binucleated Reed-Sternberg (RS) cells and the lacunar variant of RS cells, which is characterised by a folded or multilobated nucleus and lies within an open space. The background contains inflammatory cells including small lymphoctyes, plasma cells, and eosinophils. Typically, RS cells have a characteristic immunophenotype, being positive for PAX5 (Bcell transcription factor), CD15, and CD30. However, HL may lack the inflammatory background focally, and multinucleated RS cells can be prominent and may be seen in clusters, highly resembling other malignancies such as choriocarcinoma.

The cytology of the patient's transbronchial aspirate from his suspected second relapse demonstrated similarities between choriocarcinoma and HL cells (ig. 1 ). The resected lesion with cells resembling RS cells was demonstrated by the patient's excisional lymph node biopsy ( fig. 2). However, upon histochemical staining, positive staining for the cytokeratin marker CKCAM5.2 (ig. 3 ) confirmed the diagnosis of choriocarcinoma. As described, on autopsy the tumour was found to be in the mediastinum, invading adjacent structures and organs. The metastatic deposits in both intra- and extrathoracic locations had histological features identical to those of the primary tumour. It is interesting that no residual thymic parenchyma was identified in the tumour margins.

It is known that patients diagnosed with HL who are treated with chemotherapy and radiation therapy have an increased long-term risk of developing a secondary malignancy. Late relapses of HL are also possible but rare, and hence should prompt suspicion of another malignancy. Myelodysplastic syndrome, acute myeloid leukemia, and lung cancer make up the majority of cases, but the list of possible secondary cancers also includes non-Hodgkin lymphoma, breast cancer, gastric cancer, sarcoma, and melanoma. The major risk of developing a secondary solid tumour is attributable to radiation therapy, which has also been linked to pulmonary fibrosis and accelerated atherosclerosis. Alkylating chemotherapeutic drugs seem to be responsible for the increased risk of acute myeloid leukemia and myelodysplastic syndrome. However, this is the first case, to our knowledge, of a mediastinal choriocarcinoma arising after treatment for HL and masquerading as relapsed disease.

\section{Conclusions}

We describe the first case in which mediastinal choriocarcinoma developed after treatment for HL. We bring attention to this extremely rare malignancy that can masquerade as HL clinically and morphologically, and highly encourage prompt, 
adequate tissue biopsy sampling with immunohistochemical studies to confirm this rare and elusive diagnosis. Currently, the consensus is to use a cisplatin-based chemotherapy for treatment, but further research will need to be done to evaluate which chemotherapeutic regimens would be the most effective for the small group of patients with this diagnosis.

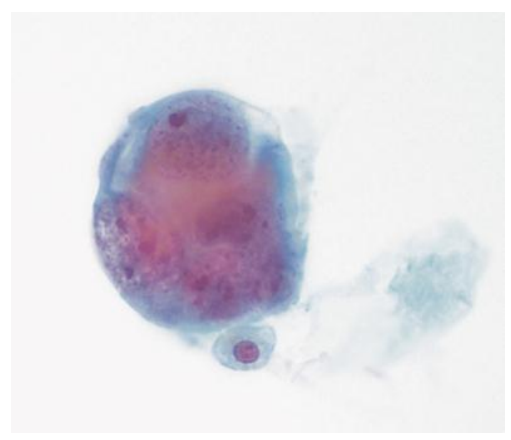

Fig. 1. Cytology of the patient's transbronchial aspirate from his suspected second relapse.

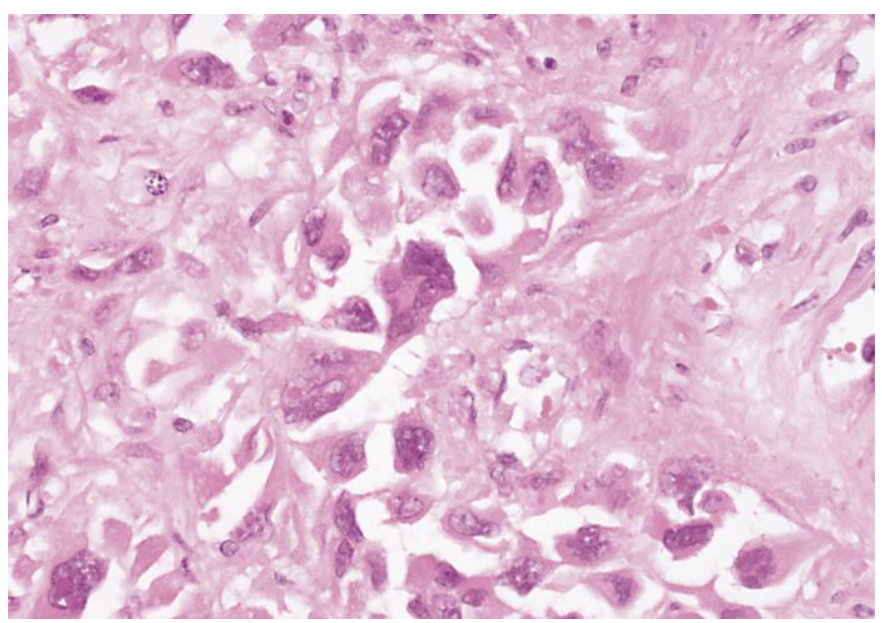

Fig. 2. Excisional lymph node biopsy with cells resembling RS cells. 


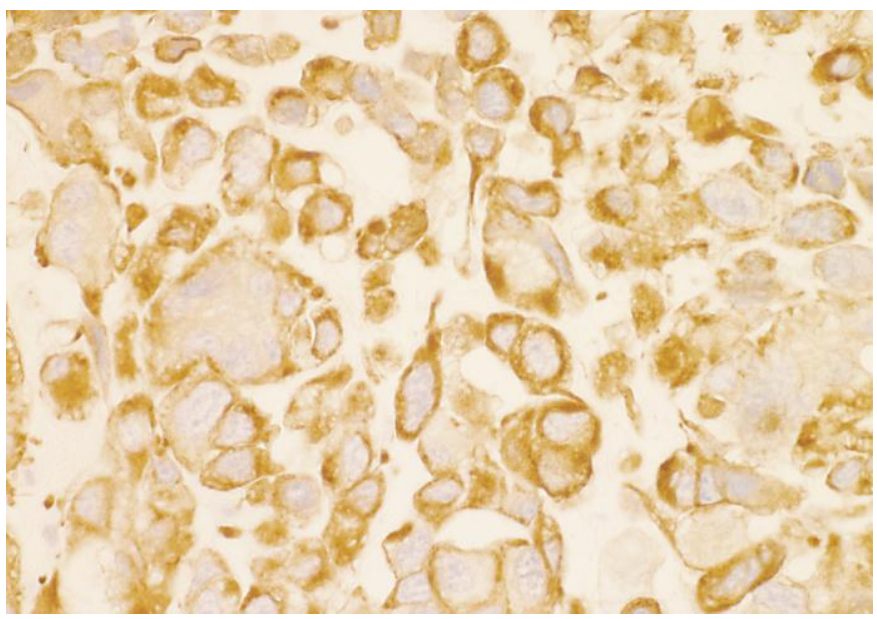

Fig. 3. Excisional lymph node biopsy demonstrating positive staining for the cytokeratin marker CKCAM5.2.

\section{References}

1 Aliotta PJ, Castillo J, Englander LS, Nseyo UO, Huben RP: Primary mediastinal germ cell tumours. Histologic patterns of treatment failures at autopsy. Cancer 1988;62:982-984.

-2 Matsutani M, Takakura K, Sano K: Primary intracranial germ cell tumours: pathology and treatment. Prog Exp Tumour Res 1987;30:307-312.

3 Martini N, Golbey RB, Hajdu SI, Whitmore WF, Beattie EJ Jr: Primary mediastinal germ cell tumours. Cancer 1974;33:763-769.

-4 Saltzman B, Reid Pitts W, Darracott Vaughan E: Extragonadal retroperitoneal germ cell tumours without apparent testicular involvement: a search for the source. Urology 1986;27:504-507.

5 Inoue H, Iwasaki M, Ogawa J, et al: Pure yolk-sac tumour of the lung. Thorac Cardiovasc Surg 1993;41:249-251.

-6 Contreras AL, Punar M, Tamboli P, Tu SM, Pisters L, Moran C, Czerniak BA, Guo CC: Mediastinal germ cell tumours with an angiosarcomatous component: a report of 12 cases. Hum Pathol 2010;41:832-837.

7 Moran, CA, Suster S: Primary germ cell tumours of the mediastinum. I. Analysis of 322 cases with special emphasis on teratomatous lesions and a proposal of histopathologic classification and clinical staging. Cancer 1997;80:681-690.

8 Moran CA, Suster S: Primary mediastinal choriocarcinomas: a clinicopathologic and immunohistochemical study of eight cases. Am J Surg Path 1997;21:1007-1012. 